\title{
MR and CT Techniques
}

\author{
João Lopes Dias and Teresa Margarida Cunha
}

\section{Contents}

1 Introduction. 000

2 Magnetic Resonance Imaging . 000

$2.1 \quad$ Introduction.................................................... 000

2.2 Patient Preparation and Positioning .................. 000

2.3 Coils, Scan Planes, and General Protocols ..... 000

2.4 Gadolinium-Based Contrast Media................ 000

3 CT Technique ........................................... 000

3.1 Introduction..................................................... 000

3.2 Technical Disadvantages................................ 000

3.3 Patient Preparation and Positioning ................ 000

3.4 Oral and Rectal Contrast............................... 000

3.5 Intravenous Iodine-Based Contrast Media...... 000

References 000

\begin{abstract}
Magnetic resonance imaging (MRI) and computed tomography (CT) are routinely used in female pelvis imaging. MRI is primarily useful for locoregional characterization of benign and malignant diseases. CT is less accurate in locoregional evaluation, but remains useful in the follow-up of treated gynecological malignancies, as well as in the setting of emergency and in the guidance of biopsies. Although transabdominal and transvaginal ultrasonography (US) is not under the scope of this chapter, it remains the first-line imaging method for most gynecological conditions.
\end{abstract}

J. Lopes Dias $(\bowtie)$

Centro Hospitalar de Lisboa Central, Lisbon, Portugal

Hospital Lusíadas de Lisboa, Lisbon, Portugal

New Medical School, Lisbon, Portugal

e-mail: joaolopesdias85@gmail.com

T.M. Cunha

Instituto Português de Oncologia de Lisboa Francisco

Gentil, Lisbon, Portugal

e-mail: tmargarida@gmail.com

\section{Introduction}

Magnetic resonance imaging (MRI) and computed tomography (CT) are routinely used in female pelvis imaging. MRI has a higher softtissue contrast and allows an accurate anatomic characterization of the pelvis as a whole, and a detailed depiction of the zonal anatomy. Thus, it is primarily useful for locoregional characterization of benign and malignant diseases. CT is less accurate in locoregional evaluation, but remains useful in the follow-up of treated gynecological malignancies, as well as in the setting of emergency (assessment of postsurgical complications and 
pelvic infectious diseases) and in the guidance of biopsies. Although transabdominal and transvaginal ultrasonography (US) is not under the scope of this chapter, it remains the first-line imaging method for most gynecological conditions.

This chapter aims to focus on some important, hands-on topics regarding MRI and CT techniques, trying not to exhaustively develop issues with isolated historical interest.

\section{Magnetic Resonance Imaging}

\section{$2.1 \quad$ Introduction}

The introduction of modern phased-array coils with eight or more elements led to high signal-tonoise ratios (SNR) and consequently increased image quality. Additionally, pelvic examinations have become faster as turbo (TSE) and fast spinecho (FSE) sequences have replaced conventional spin-echo (SE) sequences. Most MRI examinations are now performed at 1.5 and 3 Tesla (T) magnets. Despite significant improvement on SNR with higher field strength, 3 T magnets are more prone to magnetic susceptibility artifacts, which may be particularly prominent on diffusion-weighted imaging (DWI).

\subsection{Patient Preparation and Positioning}

Before performing an MR exam, the patient should be informed about its approximate duration as well as the necessity to use earplugs to protect against loud noises and to place a surface coil close to the skin. Moreover, all patients should be asked about contraindications and claustrophobia. Sedation may be required for those patients who would really benefit from the examination, but are unable to proceed due to claustrophobia. Any implanted device must be previously known and considered to be safe for the patient undergoing an MR procedure. Intrauterine devices (IUD) can be normally scanned, and usually appear as a hypointense linear structure within the endometrial cavity on both T2-weighted images (T2WI).

Finally, patients should also be informed about the utility of intravenous (IV) contrast and spasmolytic agent administration, as well as their side effects (see next sections).

Whenever a pregnant woman is undergoing an MR exam, issues about the fetus development are raised. As a general rule, the risk-benefit ratio should be evaluated for every patient. Fetal deleterious effects have not been documented on 1.5 T magnets. However, some experts still recommend avoiding the exam in the first trimester unless the potential benefits compensate the hypothetical risks. Since most of the studies were performed on 1.5 T scanners, far less is known about potential effects on $3 \mathrm{~T}$ (Masselli et al. 2013; Ray et al. 2016).

Some authors advocate that patients should void about $1 \mathrm{~h}$ before the examination to ensure that the bladder is only moderately filled. A full bladder may hamper T2WI and give rise to motion artifacts due to patient discomfort.

A 4-h fast helps to reduce bowel peristalsis and is recommended in some centers when intravenous contrast administration is required. The administration of a fast-acting laxative enema to clean the bowel may also improve image quality.

It is uncommon to perform bowel preparation with diluted barium sulfate or other solutions as it increases preparation and imaging time and does not seem to bring significant advantages.

Vaginal tampon should be avoided. Vaginal opacification with ultrasound gel makes the evaluation of vaginal walls easy and may be recommended when studying vagina tumors and deep endometriosis. Moreover, rectal and/or vaginal opacification with ultrasound gel may be useful in dynamic pelvic floor and deep endometriosis studies (Beddy et al. 2012; Sala et al. 2013; Bazot et al. 2016).

Female pelvis imaging is usually performed with patients in supine position with the arms placed by their side. The placement of a bolster 
under the knees makes the examination more comfortable. Two important procedures are generally taken in order to decrease motion artifacts during abdominal and pelvic examinations: the use of a belt covering phased-array body coils, which restricts respiratory excursions, and the application of a spatial presaturation slab in a sagittal scout view, which is particularly useful in sequences without breathhold, because of its ability to reduce artifacts from the movement of the anterior abdominal wall (Sala et al. 2013; Forstner et al. 2016; Froehlich et al. 2009).

\subsubsection{Spasmolytic Medication}

The administration of antispasmodic agents such as scopolamine $N$-butyl bromide (Buscopan ${ }^{\circledR}$ ) or glucagon is indicated to reduce artifacts from small bowel and bladder motion. It is particularly useful in the assessment of peritoneal implants on both morphological and functional sequences, especially on DWI. The administration of intravenous Buscopan ${ }^{\circledR}$ (20-40 mg) immediately before the examination is the most consensual option. Longer examinations may justify a second identical dose, because IV Buscopan $^{\circledR}$ action only lasts about $15 \mathrm{~min}$. Intramuscular (IM) administration (20 mg) has an increased length of action (approximately 30-60 min). This anticholinergic drug should not be administered in patients who have demonstrated prior hypersensitivity to scopolamine $\mathrm{N}$-butyl bromide, as well as in those with myasthenia gravis, narrow-angle glaucoma, megacolon, tachycardia, prostatic enlargement with urinary retention, paralytic ileus, or mechanical stenosis in the gastrointestinal tract. Data regarding contraindications during pregnancy is scarce; therefore Buscopan ${ }^{\circledR}$ is not recommended. Common undesirable effects may be accommodation disorders, tachycardia, dizziness, or dry mouth. If accommodation changes occur, patients should be advised not to drive.

When Buscopan ${ }^{\circledR}$ is contraindicated, glucagon (1 mg) can be administrated intravenously (Beddy et al. 2012; Sala et al. 2013; Bazot et al. 2016). In a study of Froehlich et al., glucagon had more reliable onset of action and induced longer bowel paralysis when compared to scopolamine $N$-butyl bromide. Glucagon is contraindicated in patients with known hypersensitivity to the substance, as well as in those with known pheochromocytoma (due to the risk of stimulating catecholamine release). In patients with a known insulinoma, glucagon should be administered cautiously since its initial hyperglycemic effect may stimulate the release of insulin and cause subsequent hypoglycemia (Froehlich et al. 2009).

\subsection{Coils, Scan Planes, and General Protocols}

Female pelvic MRI is generally performed with a phased-array body coil with at least four elements. The introduction of modern coils improved SNR and allowed parallel imaging, thus reducing scan time on $\mathrm{T} 1 \mathrm{~W}$ and $\mathrm{T} 2 \mathrm{~W}$ conventional sequences. Intracavitary coils, either endovaginal or endorectal, have no current scientific support (Sala et al. 2013; Allen et al. 2014).

A general female pelvis protocol usually begins with a coronal localizer, which provides an anatomic overview of both lower abdomen and pelvis. It is helpful not only to guide pelvic sequences but also to exclude other conditions like hydronephrosis or renal malformations. Fast sequences like single-shot turbo or fast spin echo are generally used.

Specific protocols depend on the study target, but usually include an axial T1WI sequence and at least two T2WI sequences in different planes (Fig. 1). Dynamic contrast-enhanced magnetic resonance imaging (DCE-MRI) and DWI have become part of the standard imaging protocols for most of female pelvis MR examinations (Forstner et al. 2010, 2016; Allen et al. 2014; Sala et al. 2011).

Table 1 resumes a suitable general protocol for gynecological MRI. Specific parameters should always be adapted according to the magnet and coils available in each center. 

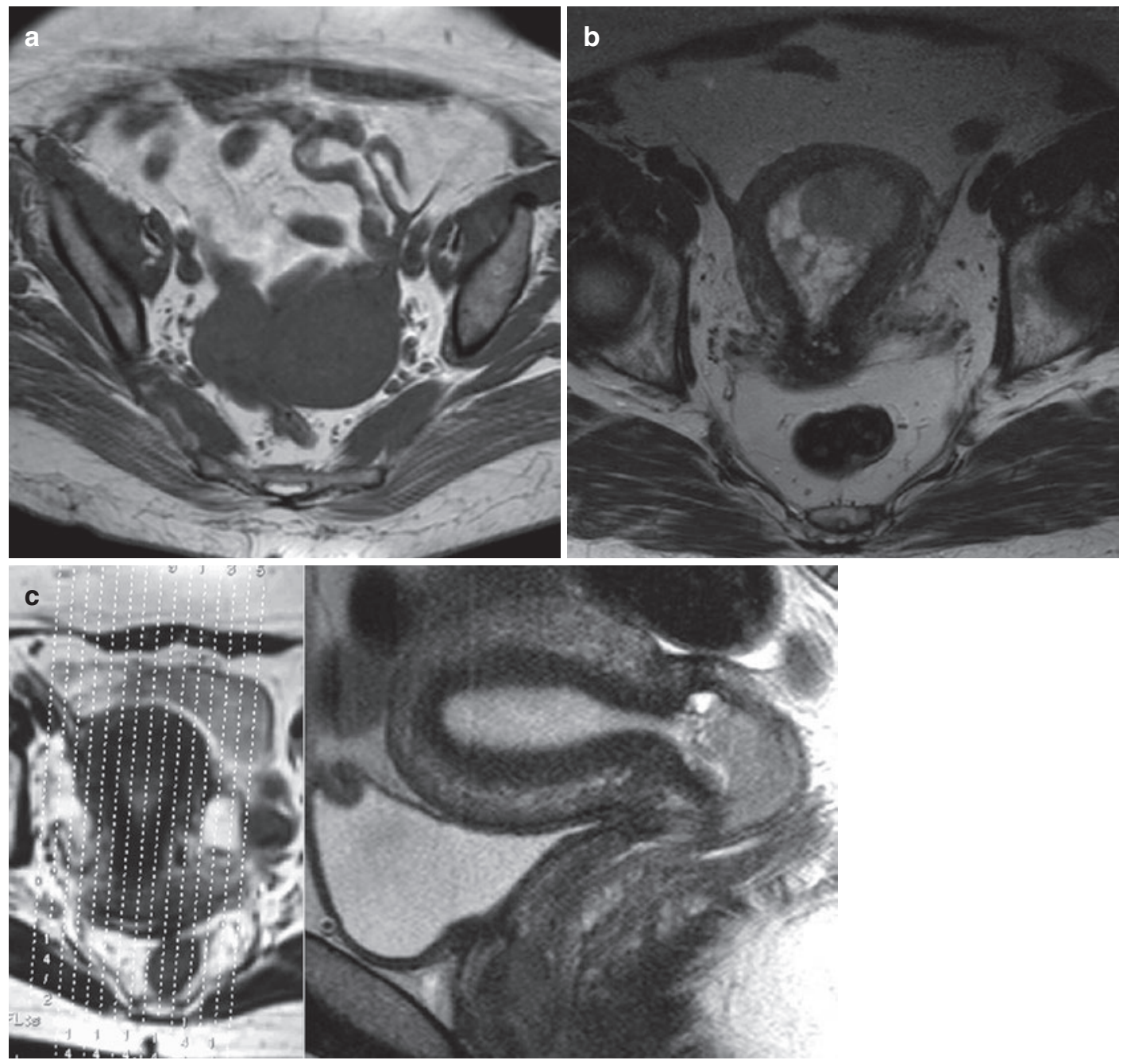

Fig. 1 Examples of different orientations on MRI in distinct patients. Axial T1W image of the pelvis (a). Axial T2W image through the pelvis showing the uterus with an endometrial carcinoma associated with a polyp (b). Oblique sagittal according to the uterine axis T2W image showing the normal uterine zonal anatomy and a cervical cancer (c). Oblique coronal according to the long axis of the uterus, parallel to the endometrial cavity (d). Axial oblique T2W image, perpendicular to the long axis of the cervix, for evaluation of parametrial invasion while staging a cervical cancer (e). Axial oblique TIW images after gadolinium with fat saturation, perpendicular to the long axis of the uterus, for local staging of an endometrial can$\operatorname{cer}(\mathbf{f})$ 

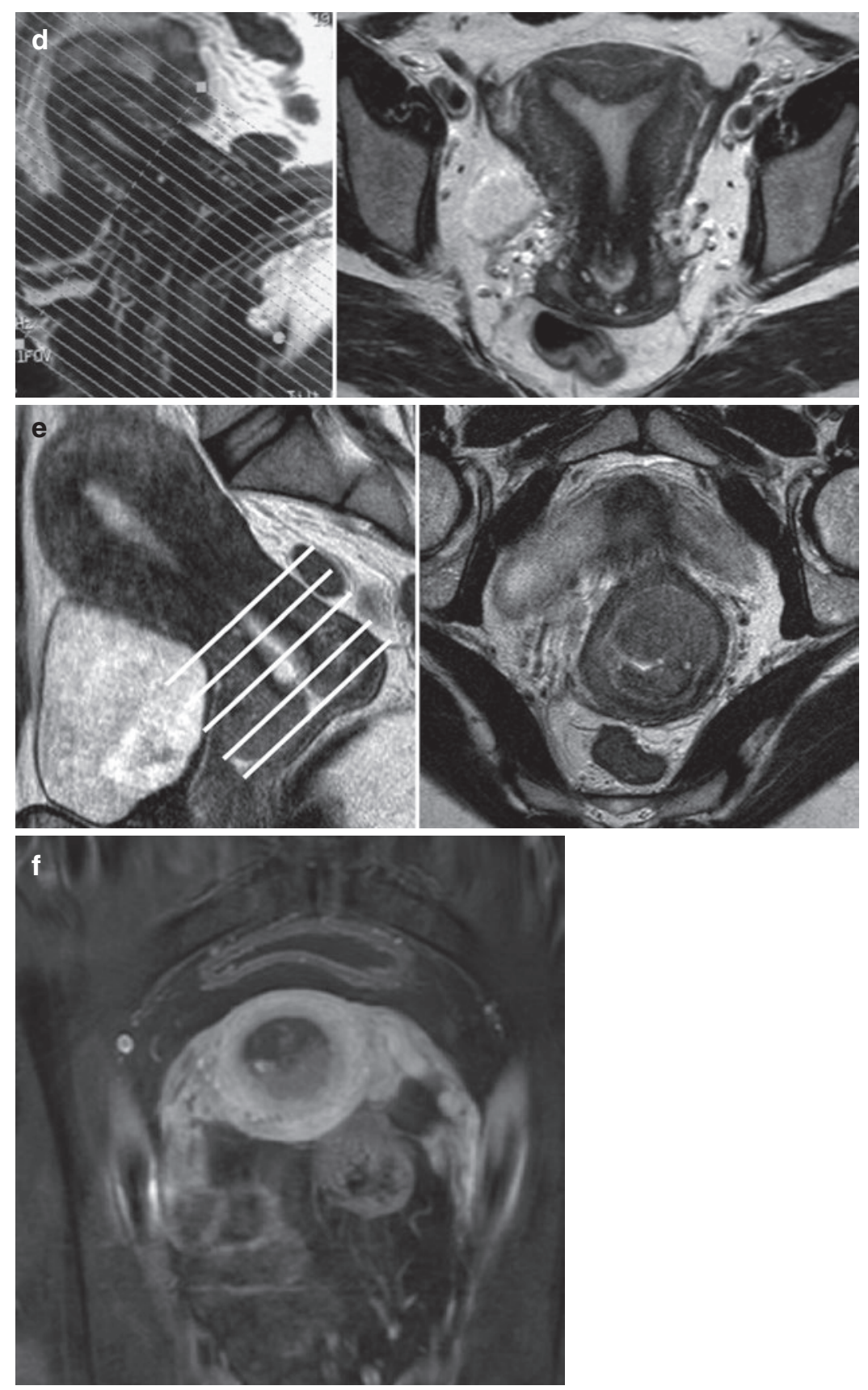

Fig. 1 (continued) 
Table 1 Suitable general protocol for gynecological MRI at a $1.5 \mathrm{~T}$ magnet

- 4-6 h of fasting

- Voiding and ingestion of two glasses of water 1 to $2 \mathrm{~h}$ before the exam

- Avoid the use of vaginal tampon

- Vaginal opacification with ultrasound gel - May be useful for vaginal tumors and deep endometriosis

- Rectal and/or vaginal opacification with ultrasound gel

- May be useful for dynamic pelvic floor studies and deep endometriosis

- Administration of a fast-acting laxative enema to clean the bowel, one on the day before the exam and another during the morning of the exam (optional)

- Supine position

- Pelvic phased-array coils with at least four elements

- Belt covering phased-array body coils

- Anterior and superior saturation band

- Antispasmodic agents:

- Buscopan 20/40 mg IM/IV

- Glucagon $1 \mathrm{mg} \mathrm{IV}$

- Matrix: $512 \times 512$

- Coronal localizer: single-shot turbo or fast spin-echo

- Morphological sequences: axial T1WI and at least two different planes on T2WI (or single 3D T2WI with posterior multiplanar post processing)

- DWI: field-of-view and thickness equal to T2WI (preferentially axial)

- Pelvis (b values: 0, 600 and $1000 \mathrm{~s} / \mathrm{mm}^{2}$ )

- Abdomen (b values: 0, 500 and $1000 \mathrm{~s} / \mathrm{mm}^{2}$ )

- High b values may reach 1200-1400 s/mm²

- DCE-MRI: 2D or 3D fat-suppressed T1W GRE sequence

- Scan plane, region of interest, and acquisition timing depending on the specific target of the study

- Gadolinium standard dose: $0.1 \mathrm{mmol} \mathrm{Gd}$ / kg body weigh

\subsubsection{T1- and T2-Weighted Imaging}

Typical SE T1WI is usually performed to assess spontaneous hyperintense content that may correspond to fat or blood. Axial fat-saturated T1WI in the same plane and with the same thickness should be performed for their distinction. Some centers perform T1W in- and opposed-phase images, which allow the detection of intravoxel lipid within masses (manifesting as a signal loss on the opposed-phase images). If a short scan time is needed, gradient-echo (GRE) sequences may be applied; however anatomic detail will be decreased. Fat saturation may also be achieved by the Dixon method, a chemical shift-based technique that acquires both in- and opposedphase images simultaneously, thus allowing mathematical combinations into fat-only and water-only sequences. It results in a more uniform suppression of the fat signal, and allows the detection and quantification of microscopic lipid (Allen et al. 2014).

Multiplanar high-resolution nonfat-saturated $\mathrm{T} 2 \mathrm{~W}$ sequences are the most relevant for the majority of female pelvic diseases, because of their ability to depict uterine and ovarian zonal anatomy and to provide good contrast between normal and pathological tissues. TSE or FSE sequences are typically used. A single 3D T2W sequence with later multiplanar postprocessing can alternatively be performed. Single-shot TSE sequences during breath-hold (HASTE) may be useful in the coronal plane to exclude hydronephrosis or to assess renal malformations.

Fat-suppressed T2W sequences are not routinely used for female pelvic imaging. However, they may be helpful to identify intraperitoneal, extraperitoneal, and endoluminal fluid; enlarged lymph nodes; and bone changes (for example, in the setting of bone metastases or of edematous changes due to postradiotherapy insufficiency fractures). When compared to nonfat-suppressed T2WI, it also helps to assess for macroscopic lipid content (Allen et al. 2014).

\subsubsection{Diffusion-Weighted Imaging}

DWI is a functional MR technique that assesses the random movement or the Brownian motion of water molecules in different physical media. The diffusion properties of a biological tissue are related to the amount of interstitial free water and permeability, therefore reflecting tissue cellularity and presence of intact cellular membranes. In general, high-cellularity tumors show restricted diffusion when compared to normal tissue, because of their higher cellular density. Coagulative necrosis, highly viscous fluid, and 
abscesses may behave similarly. DWI is performed using two or more $b$ values (a measure of the gradient strength), including one or more low $b$ values $\left(0\right.$ or $\left.50 \mathrm{~s} / \mathrm{mm}^{2}\right)$ and a high $b$ value $\left(1000 \mathrm{~s} / \mathrm{mm}^{2}\right.$ or higher). Apparent diffusion coefficients (ADC) are mathematical transformations of $b$ value acquisitions that represent the slope of the line of the natural logarithm of signal intensity ( $y$-axis) versus $b$ values ( $x$-axis). ADC maps are displayed parametrically as grayscale images. Suspicious areas with true water molecule movement restriction appear bright at high $b$ values and dark on the ADC map. High $b$ value sequences and the ADC map should always be interpreted together with morphological sequences to avoid potential pitfalls. In order to facilitate this evaluation, fusion images between T2WI and DWI may be generated. However, they can be altered by patient motion and bladder distention during the examination, which may change the relative position of pelvic organs.

Whole-body diffusion-weighted MRI protocols have been developed over the last years, mainly for cancer staging and follow-up. Shorttime examinations are now possible on both 1.5and 3-T magnets due to the evolution of echo-planar and parallel imaging, generation of high-performance gradients, and introduction of phased-array multichannel surface coils. Advantages include absence of ionizing radiation and no injection of isotopes or intravenous contrast media (Sala et al. 2013; Whittaker et al. 2009; Hameeduddin and Sahdev 2015; Qayyum 2009).

\subsubsection{Dynamic Contrast Enhancement}

Gadolinium-based contrast agents act by shortening $\mathrm{T} 1$ relaxation time, which is better seen on T1WI. Thus, dynamic contrast-enhanced MRI (DCE-MRI) is usually performed using a 2D or 3D fat-suppressed T1W GRE sequence. 3D sequences like volumetric interpolated breathhold examination (VIBE) allow the acquisition of thinner slices. The injection should be preferentially performed using an MR-compatible auto- matic injector, but manual administration may also be performed. Serial image acquisitions are then performed every few seconds over a length of a few minutes. The protocol-including scan plane, region of interest, and acquisition timewill depend on the specific target of the study (see appropriate chapters). Qualitative, semiquantitative, and quantitative analysis may be performed. The enhancement of a given structure may be directly and qualitatively accessed as an area of increased signal intensity (SI) on T1WI. Semiquantitative analysis implies recording the SI of a region of interest before and after contrast administration in order to get dynamic time-signal intensity (TSI) curves, which enable the extraction of some data like time to onset of enhancement, relative signal intensity, maximum postcontrast SI-to-precontrast SI ratio, rate of enhancement, and area under the curve (overall enhancement). Quantitative analysis-namely Ktrans (volume transfer constant between the plasma and the extracellular extravascular space)—remains under study and is not currently available in many centers (Beddy et al. 2012; Hameeduddin and Sahdev 2015; Bernardin et al. 2012).

Initial unenhanced imaging is helpful to detect hyperintense hemorrhagic or proteinaceous content, and also allows performing subtraction imaging, which requires cautious breath-holding instructions to guarantee similar registration between unenhanced and contrast-enhanced images. Regardless of those instructions, some artifacts may be found due to bladder filling during the examination.

\subsection{Gadolinium-Based Contrast Media}

A standard dose of $0.1 \mathrm{mmol} / \mathrm{kg}$ body weight of gadolinium is typically administered. For MR angiography, it may be increased to $0.2 \mathrm{mmol} / \mathrm{kg}$ body weight. The risk of nephrotoxicity is very low when gadolinium-based contrast media are used in approved doses (Beckett et al. 2015). 
The risk of an acute reaction to a gadolinium-based contrast agent is low when compared to iodine-based contrast agents. However, similar cautions should be taken. Risk patients are those with a history of previous acute reaction to gadolinium-based contrast agent, asthma, and allergy requiring medical treatment. Unlike iodine-based contrast agents, the risk of reaction to gadoliniumbased contrast agents is not related to osmolality (Beckett et al. 2015).

Nephrogenic systemic fibrosis (NSF) is recognized as a very late reaction to gadoliniumbased contrast media since 2006 . It usually starts with pain, pruritus, swelling, and erythema in the legs, and progresses to thickening of the skin and subcutaneous tissues, as well as to fibrosis of internal organs and respiratory muscles, which may lead to variable consequences ranging from contractures to cachexia and death. The severity of the disease implies the prompt recognition of high-risk patients, which are those with chronic kidney disease (CKD) 4 and 5 (GFR $<30 \mathrm{~mL} /$ min), including patients on dialysis, and those with acute kidney insufficiency. Gadoliniumbased contrast agents with higher risk of NSF are gadodiamide (DTPA-BMA), gadopentetate dimeglumine (DTPA), and gadoversetamide (DTPA-BMEA). These high-risk agents should never be given in higher doses than $0.1 \mathrm{mmol} / \mathrm{kg}$ and are contraindicated in patients with stage 4 and 5 CKDM, including those on dialysis, in patients with acute renal insufficiency, in pregnant women, and in neonates (Beckett et al. 2015; Thomsen et al. 2013, 2016; Mathur and Weinreb 2016).

Low-risk gadolinium contrast agents may be used in pregnancy when there is a strong need and no neonatal tests are necessary. Lactating women are frequently object of concern. If the above-mentioned high-risk agents are used, breastfeeding should be stopped for $24 \mathrm{~h}$ (Beckett et al. 2015; Thomsen et al. 2013, 2016; Mathur and Weinreb 2016).

\section{CT Technique}

\subsection{Introduction}

The advent of helical scanning movement and multislice data collection gave rise to a new era of fast and high-resolution acquisitions.

Modern CT scans show substantial improvement on volume coverage, scan speed, as well as a more efficient use of X-ray tubes. Helical CT scans allow near-isotropic acquisitions, thus enabling high-resolution multiplanar reconstructions (MPR) and volume rendering. Moreover, the detector size decreased and multiple arrays were incorporated inside. Therefore, several slices can be recorded simultaneously, shortening the exposure time; thus thinner slices can be obtained and partial volume artifacts can be decreased.

The high speed of current CT scanners allows a complete thoracic, abdominal, and pelvic examination in only one breath-hold. A CT pelvic acquisition is rarely performed alone. The scan is usually extended to the upper abdomen not only for staging and for the follow-up of malignant diseases but also when characterization of vascular, inflammatory, or infectious entities is needed.

By shortening the scan time, motion artifacts are also reduced and distinct phases of enhancement may be accurately obtained after intravenous contrast administration. Overall, multidetector CT (MDCT) scans yield high spatial, temporal, and contrast resolution, thus increasing diagnostic accuracy. MDCT has rapidly evolved from 4-detector row systems to 256-slice and 320-detector row CT systems. Currently, most centers use scanners with at least 8- to 16-detector rows. Submillimeter or millimeter volumetric acquisitions with subsequent reconstruction into $2-5 \mathrm{~mm}$ thick axial, sagittal, and coronal images is a suitable protocol for routine CT scans (Thomsen et al. 2016; Rydberg et al. 2000; Goldman 2007; Yitta et al. 2009) (Fig. 2). Table 2 resumes a suitable general protocol for gynecological CT scans. 

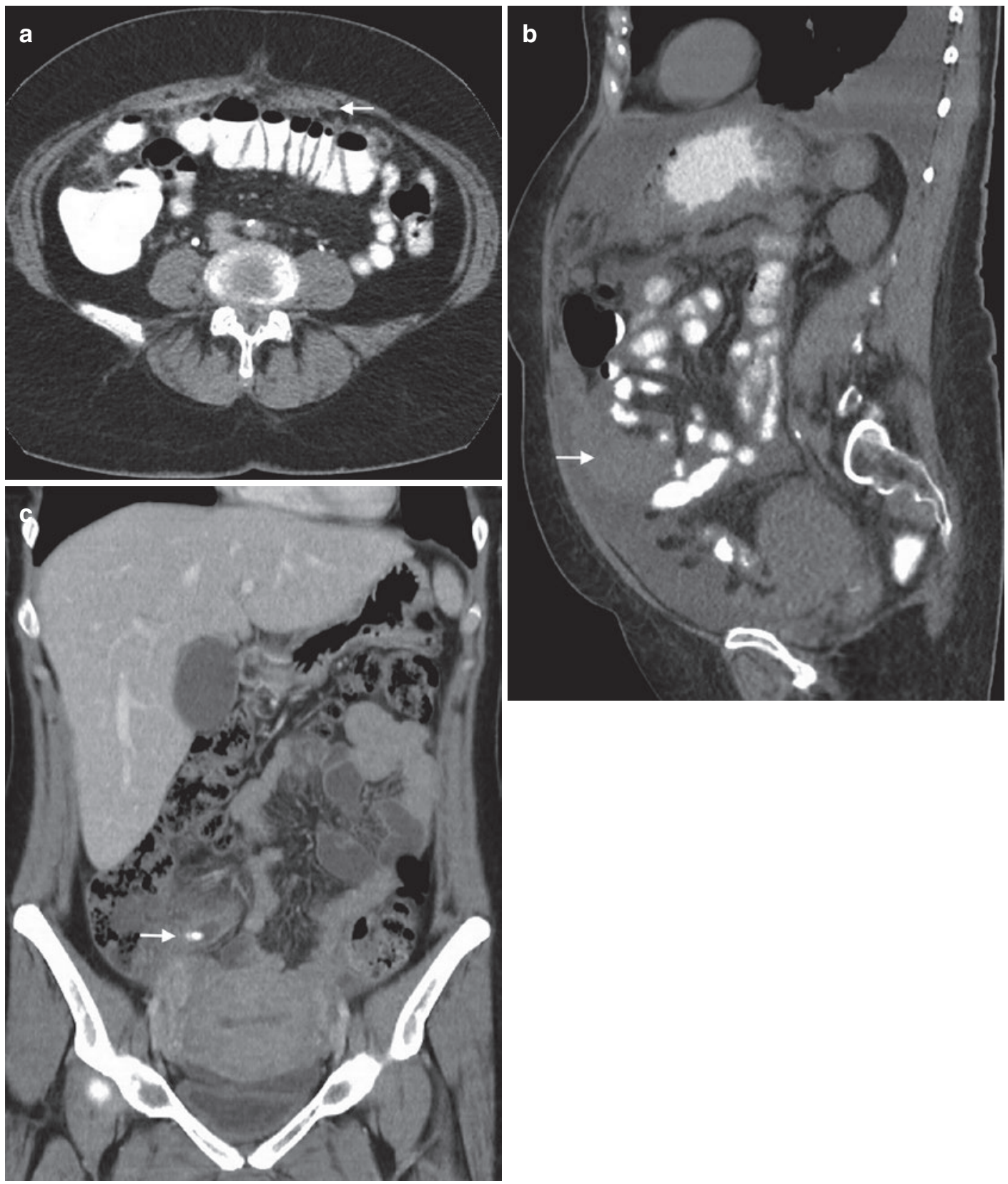

Fig. 2 Examples of different orientations on CT in three distinct patients. Axial image, after oral and intravenous contrast administration, depicting anterior peritoneal carcinomatosis (arrow) in a patient with endometrial carcinoma (a). Sagittal image, after oral contrast administration (intra-

venous contrast was contraindicated), showing ascites and huge solid peritoneal implants (arrow) in a patient with ovarian carcinoma (b). Coronal image, after intravenous contrast administration, identifying an acute appendicitis (arrow) in a young patient during early puerperium (c) 
Table 2 Suitable general protocol for gynecological CT at a 64-detector scanner

- 4-6 h of fasting

- Administration of a fast-acting laxative enema to clean the bowel, one on the day before the exam and another during the morning of the exam (optional)

- Water or iodine-based oral contrast: 1000$1500 \mathrm{~mL}$ of contrast medium administered in separate doses 45 to $60 \mathrm{~min}$ prior to the examination

- Water or iodine-based rectal contrast: catheter and enema bag; $200 \mathrm{~mL}$ to the rectosigmoid and 900-1200 mL to the entire colon

- Supine and "foot-first" positions

- Range from diaphragm to pubic bone

- Inspiration

- Scout image: $120 \mathrm{kV}, 10-50 \mathrm{~mA}$

- Final acquisition: (20 kV, 100-200 mAs)

- Sub-mm or mm volumetric acquisitions with subsequent reconstruction into 2 to $5 \mathrm{~mm}$ thick axial, sagittal and coronal images

- Correction of hip prosthesis artifacts if need: filtered back projection, adaptive filtering or iterative algorithms

- Intravenous iodine-based contrast: $100-150 \mathrm{~mL}$ at 3-4 mL/s

- Scan delay

- 70-100 s (covering all range)

- $\quad 3-5$ min (if pelvic vein thrombosis is suspected)

- 5-10 min (if bladder and ureteral opacification is needed)

\subsection{Technical Disadvantages}

Among the main disadvantages of CT, there are two with great relevance in daily practice: ionizing radiation exposure and metallic artifacts. Despite the kind of CT scan, radiation reduction and protection should always be considered. Both technicians and radiologists should be familiarized with some technical features including the tube current, X-ray beam collimation, and pitch, and cautiously select both the number and length of scan sequences. Dose quantification parameters like volume CT dose index (CTDlvol) and dose-length product (DLP) are usually displayed in current CT scans and should be routinely checked. Hip prosthesis causes substantial artifacts due to photon starvation and beam hardening, consequently hampering not only the joint and the surrounding muscles, but also pelvic organs. Methods may be used to reduce these artifacts: filtered back projection (FBP), which uses information from areas adjacent to regions affected by metal artifacts and replaces the metalcorrupted raw data by the interpolated values; adaptive filtering, which corrects the excessive noise produced by the photon starvation effect; or iterative algorithms, which use a combination of different metal artifact reduction algorithms and algebraic reconstruction techniques (Morsbach et al. 2013). Iterative reconstruction techniques also enable performing reduced-dose CT examinations (due to either tube current or tube potential lowering) without altering image quality (Padole et al. 2014).

\subsection{Patient Preparation and Positioning}

Before a CT scan, an inquiry about the patient medical history, routine medication, and potential contraindications is mandatory. Pregnancy is not a formal contraindication to CT; however, it should be avoided mainly during the first trimester. Patient preparation for CT scans is mainly related to contrast material administration (see next sections). Due to the need of intravenous contrast, patients are optimally asked to fast at least for $4 \mathrm{~h}$. Administrating a fast-acting laxative enema to clean the bowel may also be recommended.

Patients are usually scanned in supine and "foot-first" positions, with their arms raised above their heads. Claustrophobic patients tolerate lying with the head outside the gantry better. Moreover, these positions allow for a faceto-face communication with the patient and facilitate the access to the patient whenever the examination has to be interrupted. "Foot-first" positioning also eases the connection of contrast medium tubes, which can be done immediately after inserting the venous access.

\subsection{Oral and Rectal Contrast}

Oral and rectal contrast media are frequently used in abdominal and pelvic CT imaging. Classically, it is given in cases of suspected bowel perforation or of anastomosis leakage. However, opacification 
of the digestive tract is also helpful for distinguishing collapsed loops from lymph nodes, peritoneal implants, pelvic masses, and fluid collections.

Water as negative or positive iodinated solutions may be used for bowel opacification. In female pelvis imaging, positive contrast is particularly useful for staging and follow-up of ovarian and endometrial cancer. Iodinated solutions are preferable over barium suspensions due to its moderate effect on peristalsis and easier distribution along the digestive tract. Contrarily, barium suspensions are more prone to flocculate and may give rise to streak artifacts that hamper wall evaluation. Both types of contrast are usually safe, with only rare cases of mild diarrhea being reported. However, if bowel perforation is suspected, only iodinated solution should be administered due to the higher peritoneal toxicity of barium-based contrast media. As 1-2\% of oral contrast is absorbed through the gut patients who had previous moderate to severe IV contrast allergy should be managed carefully.

Protocols for oral contrast vary according to the center. Typically, $1000-1500 \mathrm{~mL}$ of contrast medium is ingested in separate doses 45-60 min prior to the examination. Some authors advocate the administration of $20 \mathrm{mg}$ metoclopramide at the beginning of the ingestion, in order to shorten patient preparation.

Rectal contrast is administered when the patient lies on the scanning table by using a catheter and an enema bag. $200 \mathrm{~mL}$ of contrast usually opacifies the rectum and the sigmoid colon adequately, whereas the entire colon may require 900$1200 \mathrm{~mL}$ of contrast. Sometimes, anal diseases like hemorrhoids and fissures hamper the introduction of the rectal tube due to pain. Special care should also be taken in patients with anal and lower third rectal carcinoma due to the risk of ulceration or perforation (Beckett et al. 2015).

\subsection{Intravenous lodine-Based Contrast Media}

Intravenous iodine-based contrast media are usually given as a rapid bolus via an IV cannula, by using a pump injector. A suitable protocol for abdominal and pelvic CT scan is to administer $100-150 \mathrm{~mL}$ of $350 \mathrm{mg}$ iodine contrast media at
3-4 mL/s. As the time to contrast material arrival and peak enhancement are affected by the choice of intravenous access sites, particularly when forearm or hand veins are used, lower flow rates are desirable (Bae 2010).

A suitable routine gynecological CT scan includes the upper abdomen and is acquired 70-100 s after contrast injection. Liver metastases from gynecological malignancies are typically hypovascular, so an arterial phase is usually unnecessary. Delayed images are recommended whenever pelvic vein thrombosis is suspected (3-5 $\mathrm{min}$ ) or bladder and ureteral opacification is needed (5-10 min).

In order to reduce the risk of an acute reaction to iodine-based contrast media, some procedures should be taken according to the European Society of Urogenital Radiology (ESUR) guidelines: a nonionic contrast medium should be used; the patient should be kept in the Radiology Department for $30 \mathrm{~min}$ after the injection; and drugs and equipment for resuscitation have to be readily available. For patients at increased risk of reaction (history of previous moderate or severe acute reaction to an iodine-based contrast agent, asthma, and allergy requiring medical treatment), an alternative imaging tool not requiring an iodine-based contrast agent should be considered. The use of premedication for allergy prevention is widely accepted, despite its underlying limited clinical evidence. The ESUR guidelines recommend oral administration of prednisolone $30 \mathrm{mg}$ (or methylprednisolone $32 \mathrm{mg}$ ), 12 and $2 \mathrm{~h}$ before contrast medium administration (Thomsen et al. 2016).

There is usually some concern regarding thyrotoxicosis, a potential very late reaction to iodine-based contrast media that usually occurs more than 1 week after injection. As a general rule, iodinated contrast media should not be given to patients with manifest hyperthyroidism. Patients at risk-those with untreated Graves' disease, or multinodular goiter and thyroid autonomy, especially if they are elderly and/or live in areas of dietary iodine deficiency-should be strictly monitored by endocrinologists after injection (Van der Molen et al. 2004).

Pregnancy and lactation are also usual sources of concern for both clinicians and radiologists. 
Iodine-based contrast media may be given to pregnant patients in extraordinary conditions, but thyroid function should be checked in the neonate during the first week following the exam. When iodine-based agents are given to lactating mothers, breastfeeding may be continued normally (Thomsen et al. 2016).

In patients with renal impairment (eGFR less $45 \mathrm{~mL} / \mathrm{min} / 1.73 \mathrm{~m}^{2}$ for intravenous administration), an alternative imaging method without iodine-based contrast media should be considered. However, if it is really needed, volume expansion should be done. According to the ESUR guidelines, intravenous normal saline, $1.0-1.5 \mathrm{~mL} / \mathrm{kg} / \mathrm{h}$, for at least $6 \mathrm{~h}$ before and after contrast medium administration is recommended. An alternative suitable protocol is intravenous sodium bicarbonate $(154 \mathrm{mEq} / \mathrm{L}$ in dextrose $5 \%$ water), $3 \mathrm{~mL} / \mathrm{kg} / \mathrm{h}$ for $1 \mathrm{~h}$ before contrast medium administration and $1 \mathrm{~mL} / \mathrm{kg} / \mathrm{h}$ for $6 \mathrm{~h}$ after contrast medium administration. Moreover, the lowest dose of a low or iso-osmolar contrast medium should be used (Thomsen et al. 2016).

Finally, some recommendations should be followed in patients taking metformin due to the risk of lactic acidosis after IV iodine-based contrast exposure when significant renal impairment is present. Patients with an eGFR between 30 and $44 \mathrm{~mL} / \mathrm{min} / 1.73 \mathrm{~m}^{2}$ (CKD 3) should stop metformin $48 \mathrm{~h}$ before contrast medium injection and should only restart it $48 \mathrm{~h}$ after the examination if renal function has not deteriorated. In patients with eGFR less than $30 \mathrm{~mL} /$ $\mathrm{min} / 1.73 \mathrm{~m}^{2}$ (CKD 4 and 5), iodine-based contrast media should be avoided (Beckett et al. 2015; Thomsen et al. 2016).

\section{References}

Allen BC, Hosseinzadeh K, Qasem SA, Varner A, Leyendecker JR (2014) Practical approach to MRI of female pelvic masses. Am J Roentgenol 202(6):13661375. doi:10.2214/AJR.13.12023

Bae KT (2010) Intravenous contrast medium administration and scan timing at CT: considerations and approaches. Radiology 256(1):32-61. doi:10.1148/ radiol.10090908

Bazot M, Bharwani N, Huchon C, Kinkel K, Cunha TM, Guerra A, Manganaro L, Buñesch L, Kido A, Togashi
K, Thomassin-Naggara I, Rockall AG (2016) European Society of Urogenital Radiology (ESUR) guidelines: MR imaging of pelvic endometriosis. Eur Radiol. doi:10.1007/s00330-016-4673-z2016

Beckett KR, Moriarity AK, Langer JM (2015) Safe use of contrast media: what the radiologist needs to know. Radiographics 35(6):1738-1750. doi:10.1148/ rg.2015150033

Beddy P, O'Neill AC, Yamamoto AK, Addley HC, Reinhold C, Sala E (2012) FIGO staging system for endometrial cancer: added benefits of MR imaging. Radiographics 32(1):241-254. doi:10.1148/rg.321115045

Bernardin L, Dilks P, Liyanage S, Miquel ME, Sahdev A, Rockall A (2012) Effectiveness of semi-quantitative multiphase dynamic contrast-enhanced MRI as a predictor of malignancy in complex adnexal masses: radiological and pathological correlation. Eur Radiol 22(4):880-890. doi:10.1007/s00330-011-2331-z

Forstner R, Sala E, Kinkel K, Spencer JA (2010) ESUR guidelines: ovarian cancer staging and follow-up. Eur Radiol 20(12):2773-2780. doi:10.1007/ s00330-010-1886-4

Forstner R, Thomassin-Naggara I, Cunha TM, Kinkel K, Marselli G, Kubik-Huch R, Spencer JA, Rockall A (2016) ESUR recommendations for MR imaging of the sonographically indeterminate adnexal mass: an update. Eur Radiol. doi:10.1007/s00330-016-4600-3

Froehlich JM, Daenzer M, von Weymarn C, Erturk SM, Zollikofer CL, Patak MA (2009) Aperistaltic effect of hyoscine $N$-butylbromide versus glucagon on the small bowel assessed by magnetic resonance imaging. Eur Radiol 19:1387-1393. doi:10.1007/ s00330-008-1293-2

Goldman LW (2007) Principles of CT and CT technology. J Nucl Med Technol 35(3):115-130. doi:10.2967/ jnmt.107.042978

Hameeduddin A, Sahdev A (2015) Diffusion-weighted imaging and dynamic contrast-enhanced MRI in assessing response and recurrent disease in gynaecological malignancies. Cancer Imaging 15:3. doi:10.1186/s40644-015-0037-1

Masselli G, Derchi L, McHugo J, Rockall A, Vock P, Weston M, Spencer J (2013) Acute abdominal and pelvic pain in pregnancy: ESUR recommendations. Eur Radiol 23:3485-3500. doi:10.1007/s00330-013-2987-7

Mathur M, Weinreb JC (2016) Imaging patients with renal impairment. Abdom Radiol 41(6):1108-1121. doi:10.1007/s00261-016-0709-8

Morsbach F, Bickelhaupt S, Wanner GA, Krauss A, Schmidt B, Alkadhi H (2013) Reduction of metal artifacts from hip prostheses on CT images of the pelvis: value of iterative reconstructions. Radiology 268(1):237-244. doi:10.1148/radiol.13122089

Padole A, Khawaja R, Kalra M, Singh S (2014) CT radiation dose and iterative reconstruction techniques. Am J Roentgenol 204:W384-W392. doi:10.2214/ AJR.14.13241

Qayyum A (2009) Diffusion-weighted imaging in the abdomen and pelvis: concepts and applications. Radiographics 29(6):1797-1810 
Ray J, Vermeulen M, Bharatha A, Montanera W, Park A (2016) Association between MRI exposure during pregnancy and fetal and childhood outcomes. JAMA 316(9):952-961. doi:10.1001/jama.2016.12126

Rydberg J, Buckwalter KA, Caldemeyer KS et al (2000) Multisection CT: scanning techniques and clinical applications. Radiographics 20(6):1787-1806. doi:10.1148/radiographics.20.6.g00nv071787

Sala E, Rockall A, Kubik-Huch R (2011) Advances in magnetic resonance imaging of endometrial cancer. Eur Radiol 21(3):468-473. doi:10.1007/s00330-010-2010-5

Sala E, Rockall AG, Freeman SJ, Mitchell DG, Reinhold C (2013) The added role of MR imaging in treatment stratification of patients with gynecologic malignancies: what the radiologist needs to know. Radiology 266(3):717-740. doi:10.1148/ radiol. 12120315

Thomsen HS, Morcos SK, Almen T et al (2013) Nephrogenic systemic fibrosis and gadolinium-based contrast media: updated ESUR contrast medium safety committee guidelines. Eur Radiol 23(2):307318. doi:10.1007/s00330-012-2597-9

Thomsen HS et al (2016) ESUR Guidelines on Contrast Media Version:9.0. http://www.esur.org/esur-guidelines/ Van der Molen AJ, Thomsen HS, Morcos SK (2004) Effect of iodinated contrast media on thyroid function in adults. Eur Radiol 14(5):902-907. doi:10.1007/ s00330-004-2238-z

Whittaker CS, Coady A, Culver L, Rustin G, Padwick M, Padhani AR (2009) Diffusion-weighted MR imaging of female pelvic tumors: a pictorial review. Radiographics 29(3):759-774. doi:10.1148/ rg.293085130discussion 774-8

Yitta S, Hecht EM, Slywotzky CM, Bennett GL (2009) Added value of multiplanar reformation in the multidetector CT evaluation of the female pelvis: a pictorial review. Radiographics 29:1987-2005. doi:10.1148/ rg.297095710 